\title{
Fatores determinantes das perdas das vacinas em Unidades Básicas de Saúde
}

\author{
Jaqueline Adelaide da Silva Santos ${ }^{1}$, Daiara Barbosa Torres ${ }^{2}$, Rosana Alves de Melo ${ }^{3}$, \\ Flávia Emília Cavalcante Valença Fernandes ${ }^{4}$, Maria Elda Alves de Lacerda Campos $^{5}$
}

\begin{abstract}
Resumo: O objetivo foi descrever os principais fatores determinantes das perdas dos imunobiológicos e o valor total dessas perdas no município de Petrolina no ano de 2013. Método: estudo descritivo, onde foram analisados 48 relatórios das 28 unidades de saúde que apresentaram perdas de imunobiológicos no período estabelecido. Resultados: foram registradas 10.439 doses perdidas totalizando um valor de $\mathrm{R} \$ 72.864,23$. A maior parte das perdas ocorreu na zona rural. A falta de energia elétrica correspondeu a 43,75\% dos motivos das perdas; $85,40 \%$ das vacinas estavam com temperatura acima do preconizado; 70,20\% permaneceram mais de 12 horas expostas à temperatura inadequada. Conclusão: $O$ estudo sinaliza a importância de conhecer as causas das perdas dos imunobiológicos, na maioria das vezes evitáveis, o que poderá subsidiar o gerenciamento nas unidades de saúde, garantindo a qualidade das vacinas que são ofertadas nos serviços de saúde.
\end{abstract}

Descritores: Vacinas, programas de imunização, refrigeração.

\section{Determinants of vaccine losses in Basic Health Units}

\begin{abstract}
The objective of this study was to describe the main determinants of immunobiological losses and the total value of these losses in the municipality of Petrolina in the year 2013. Method: descriptive study, where 48 reports were analyzed from the 28 health units that presented immunobiological losses in the Period. Results: 10,439 doses lost were recorded, totaling R \$ 72,864.23. Most of the losses occurred in the countryside. The lack of electricity corresponded to $43.75 \%$ of the reasons for the losses; $85.40 \%$ of the vaccines were at a temperature higher than recommended; $70.20 \%$ remained more than 12 hours exposed to inadequate temperature. Conclusion: The study indicates the importance of knowing the causes of immunobiological losses, which are often avoidable, which may subsidize management in health units, guaranteeing the quality of the vaccines that are offered in health services.
\end{abstract}

Descriptors: Vaccines, immunization programs, refrigeration.

\footnotetext{
${ }^{1}$ Enfermeira pela Universidade de Pernambuco - Campus Petrolina. Pós-Graduanda em Obstetrícia pela Instituto Nacional de Ensino de São Paulo (INESP). E-mail: jaqueline_adesantos@hotmail.com;

${ }^{2}$ Enfermeira pela Universidade de Pernambuco - Campus Petrolina. E-mail: daiaratorres2008@ hotmail.com;

${ }^{3}$ Doutoranda pelo Programa de Pós-graduação em Inovação Terapêutica da Universidade Federal de Pernambuco (PPGIT/UFPE). Mestrado em Enfermagem pela Universidade Estadual de Feira de Santana. Professora Assistente da Universidade de Pernambuco Campus Petrolina. Email: rosananurse@hotmail.com;

${ }^{4}$ Doutoranda pelo Programa de Pós-graduação em Inovação Terapêutica da Universidade Federal de Pernambuco (PPGIT/UFPE). Mestre em Gestão e Economia da Saúde (UFPE). Professora Assistente da Universidade de Pernambuco Campus Petrolina. E-mail: flavia.fernandes@upe.br;

${ }^{5}$ Mestre em Vigilância sobre Saúde pela Universidade de Pernambuco. E-mail: eldalcampos@gmail.com.
} 
Id on Line Revista Multidisciplinar e de Psicoloqia

Id on Line Multidisciplinary and Psycology Journa

\section{Introdução}

As vacinas são imunobiológicos compostas por um ou mais agentes imunizantes e tem como finalidade principal a redução da morbimortalidade por doenças imunopreveníveis contribuindo para a manutenção da saúde da população (BRASIL, 2001a).

No Brasil, no ano de 1970 ocorreu a implantação do Programa Nacional de Imunização (PNI) promovida pelo Ministério da Saúde, com a finalidade de coordenar ações de vacinação que se desenvolviam, até então, com descontinuidade pelo caráter episódico e pela reduzida cobertura, a fim de proporcionar sincronia e racionalização (BRASIL, 2007a).

O PNI tem como objetivo principal a erradicação e/ou controle das doenças imunopreveníveis por meio de estratégias básicas de vacinação (BRASIL 2001b). A partir da adoção dessas estratégias no Brasil, histórias bem sucedidas de vacinação erradicaram a febre amarela urbana (1942) e a varíola (1973). Com a criação do PNI, conseguiu-se a erradicação da poliomielite (1989), o controle do tétano neonatal e acidental, a redução das formas graves de tuberculose, da difteria e da coqueluche (VICARE, 2008).

A eficácia das vacinas está relacionada a diversos aspectos, além do organismo do próprio indivíduo que necessita apresentar condições fisiológicas para desenvolver a imunidade pelo produto, aspectos como temperatura adequada, prazo de validade dentre outros também são critérios fundamentais (CUNHA; KREBS; BARROS, 2009).

É necessário manter as vacinas em condição adequada de conservação, (temperatura entre $+2^{\circ} \mathrm{e}+8^{\circ} \mathrm{C}$ ), utilizando instalações e equipamentos apropriados. Por serem produtos termolábeis, o calor acelera a inativação dos componentes dos imunobiológicos após determinado tempo expostos à temperatura fora do preconizado, e essa alteração pode ocorrer tanto pela alta como pela baixa temperatura (BRASIL, 2013).

Assim, um manuseio inadequado, um equipamento com defeito, ou falta de energia elétrica podem interromper o processo de refrigeração, se não tomadas às medidas necessárias nessas intercorrências, ocorre o comprometimento na potência e na eficácia, levando os imunobiológicos a serem colocados sob suspeita ou até mesmo a perdas dos mesmos(BRASIL, 2001b)

Considerando a importância das ações de imunização visando evitar as doenças imunopreveníveis e melhorar a saúde da população, é essencial o cuidado na conservação dos imunobiológicos garantido o poder imunogênico das vacinas. É relevante conhecer as causas 
Id on Line Revista Multidisciplinar e de Psicoloqia

Id on Line Multidisciplinary and Psycology Journal

das perdas, na maioria das vezes evitáveis, de imunobiológicos, tanto para a análise do processo de trabalho nesse domínio, quanto para subsidiar o gerenciamento nas unidades de saúde, buscando minimizar o impacto dessas perdas.

Diante do exposto, o presente estudo teve como objetivo descrever os principais fatores determinantes das perdas dos imunobiológicos e o valor total dessas perdas no município de Petrolina no ano de 2013.

\section{Método}

O desenvolvimento desse trabalho foi fundamentado pela realização de um estudo descritivo de caráter documental com abordagem quantitativa, a partir da avaliação de relatórios estaduais disponíveis no PNI da VIII Gerência Regional de Saúde (VIII GERES) do estado de Pernambuco sobre descrição das perdas de imunobiológicos e seus fatores determinantes nas Unidades Básicas de Saúde (UBS) no município de Petrolina no período de janeiro a dezembro de 2013.

O município de Petrolina, localizado no estado de Pernambuco, possui 44 Unidades de Saúde com salas de vacinas, localizadas nas zonas urbana e rural, das quais 10 são unidades de Atendimento Multiprofissional Especializado (AME) sendo composta de três a quatro equipes de saúde da família ${ }^{7}$

Foram estudados 48 relatórios das 28 unidades de saúde onde apresentaram perdas de imunobiológicos no período estabelecido para a pesquisa. Ressalta-se que algumas unidades de saúde apresentaram mais de um relatório no ano de 2013.

Foram excluídos todos os relatórios que não correspondiam ao município de Petrolina, os que não se encontravam no período determinado para pesquisa e aqueles que não foram avaliados pelos técnicos do nível central da Secretaria Estadual de Saúde de Pernambuco- SESPE.

Os dados foram obtidos por meio de um formulário elaborado pelas pesquisadoras. Foram estudadas as seguintes variáveis: identificação da UBS, endereço, zona localizada, data da notificação das perdas do imunobiológico e fatores determinantes das perdas (falta de energia elétrica, falha no equipamento de refrigeração, tempo de exposição à temperatura 
Id on Line Revista Multidisciplinar e de Psicoloqia

Id on Line Multidisciplinary and Psycology Journal

inadequada, temperatura abaixo e/ou acima do estabelecido, acondicionamento inadequado, outros tipos de perdas).

Além das variáveis citadas, foram descritos, em dias, o tempo-resposta da Secretaria Estadual de Saúde de Pernambuco- SES-PE sobre os resultados das análises dos relatórios dos imunobiológicos que foram colocados sob suspeita e que aguardavam para serem descartados ou não, segundo a avaliação do PNI estadual. O total de vacinas perdidas no período estudado também foi avaliado.

O tratamento e análise dos dados foram realizados através de medidas de tendência central e dispersão e em frequências absolutas e relativas com avaliação do intervalo de confiança (95\%) para média das variáveis: tempo de exposição, tempo de resposta, total de doses perdidas e valor total. Foi utilizado o programa Microsoft Excel 2013 e os programas estatísticos gretl versão 2015d e Simple Interactive Statistical Analysis (SISA) para registro e análise dos dados respectivamente. Foi utilizado o teste estatístico Exato de Fisher para verificar diferenças significantes entre as variáveis devido aos dados apresentarem valores esperados menores que 5. Para o teste foram rejeitadas as hipóteses nulas ao nível de significância de 5\% $(\mathrm{p}<0,05)$.

Foi categorizado o tempo de exposição à temperatura inadequada da seguinte forma: de 3 horas a 12 horas e acima de 12 horas de exposição. Foi considerado o tempo mínimo encontrado de exposição para definição do início do intervalo. O número de doses também foi categorizado de 01 a 200 doses perdidas e acima de 200 doses perdidas.

O projeto de Pesquisa foi aprovado pelo Comitê de Ética em Pesquisa com Seres Humanos da Universidade de Pernambuco sob o ${ }^{\circ} 34873214.1 .0000 .5207$ e autorizado pela VIII Gerência Regional de Saúde.

\section{Resultados}

Foram avaliados 48 relatórios referentes a 28 unidades de saúde, que apresentaram perdas de imunobiológicos no município de Petrolina, no período de janeiro a dezembro de 2013. Os meses que obtiveram maior número de relatórios de perdas foram janeiro e abril, com $25,00 \%$ e $18,80 \%$, respectivamente (Figura 1). 
Id on Line Revista Multidisciplinar e de Psicoloqia

Id on Line Multidisciplinary and Psycology Journal

Como o mês de janeiro foi o período que mais apresentou prejuízos, observou-se que $83,30 \%$ das notificações de perdas das vacinas durante esse mês foi devido à falta de energia elétrica e todos os relatórios encontrados nesse período do ano estavam com temperaturas acima do estabelecido.

Figura 1. Distribuição de notificações de perdas das vacinas em número* e percentual segundo mês de ocorrência. Petrolina-PE, 2013.

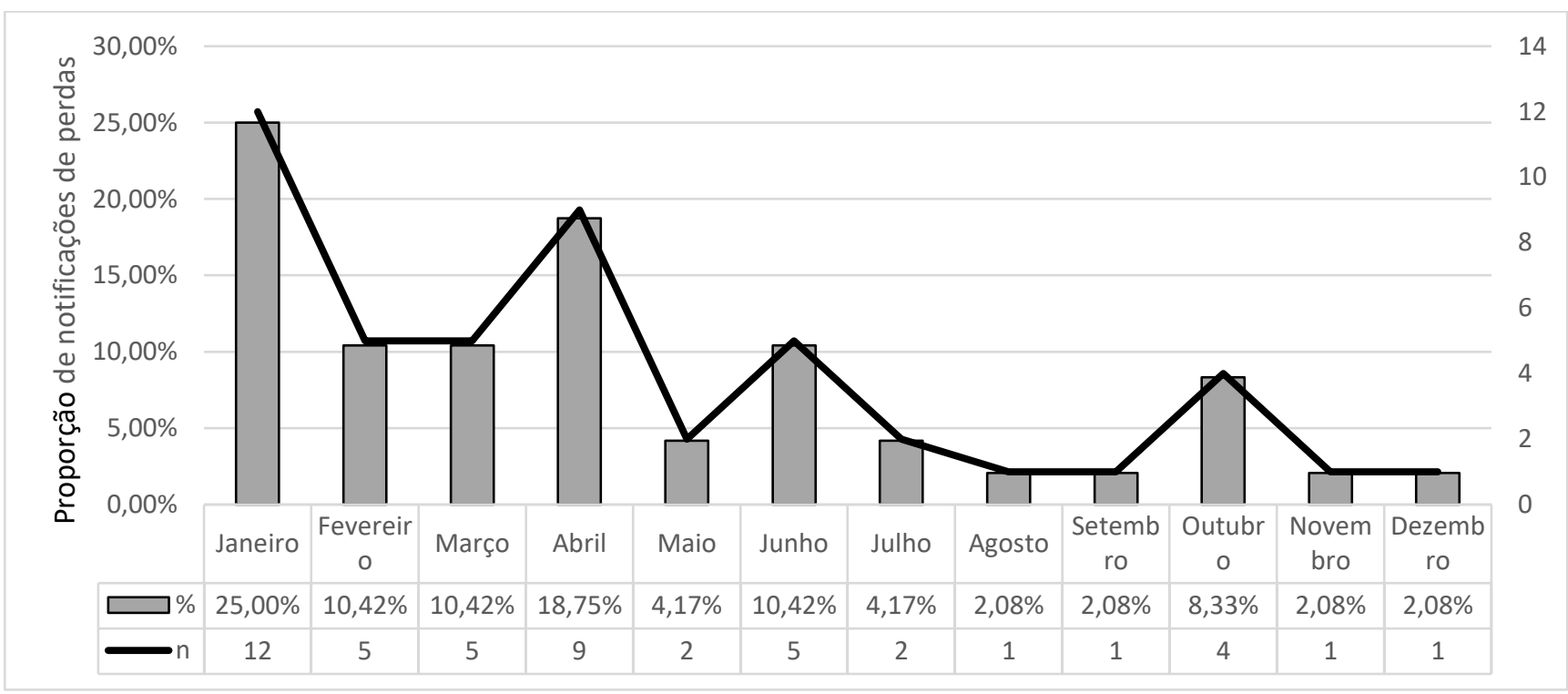

*Os números absolutos foram colocados no eixo secundário do lado direito do gráfico para melhor visualização dos dados.

Fonte: Elaboração própria a partir dos resultados da pesquisa.

Foram registradas 10.439 doses perdidas de vacinas no período estudado, totalizando um valor de $\mathrm{R} \$ 72.864,23$. Observou-se que a zona rural apresentou maior registro de relatórios de perda em relação à zona urbana respondendo por 58,30\% (28 relatórios), enquanto na zona urbana foi de 41,70\% (20 relatórios) (Tabela 1).

Considerando os fatores determinantes das perdas das vacinas no município, a falta de energia elétrica apareceu como principal causa apresentando $43,75 \%$, seguida de falha no equipamento de refrigeração com 33,33\%, acondicionamento inadequado das vacinas de $14,58 \%$ e outras causas de perdas com $8,30 \%$ (Tabela 1 ).

Comparando esses determinantes entre as zonas urbana e rural do município, considerando a hipótese nula de que os determinantes das perdas foram iguais entre as zonas de localização das unidades, observou-se que houve diferença significante $(p=0,00333)$. As 
Id on Line Revista Multidisciplinar e de Psicoloqia

Id on Line Multidisciplinary and Psycology Journal

vacinas cujas perdas estavam relacionadas a falta de energia, a maioria ocorreu na UBS da zona rural com $10(85,70 \%)$. A falha no equipamento de refrigeração e o acondicionamento inadequado das vacinas foi mais prevalente na zona urbana correspondendo a $10(62,50 \%)$ e 5 (71,40\%), respectivamente em comparação às unidades da zona rural. Outras causas que determinaram as perdas não apresentaram diferença em relação a localização da UBS (Tabela $1)$.

Observou-se que $41(85,40 \%)$ notificações encontravam-se com temperatura acima de $8^{\circ} \mathrm{C}$ e $7(14,60 \%)$ com temperatura abaixo de $2^{\circ} \mathrm{C}$. A avaliação da hipótese de que os determinantes das perdas das vacinas foram iguais entre os níveis de temperatura aos quais as vacinas estavam expostas também se apresentou significante ( $\mathrm{p}=0,01677)$. Assim, comparando os fatores causadores das perdas dos imunobiológicos com as temperaturas encontradas, percebeu-se que todos os relatórios que tinham como causa a falta de energia elétrica, as vacinas encontravam-se com temperaturas acima do preconizado pelo PNI $\left(8^{\circ} \mathrm{C}\right)$ (Tabela 1). A falha no equipamento e o acondicionamento inadequado também tiveram como consequência, na maioria dos casos, exposição a temperaturas elevadas, $11(68,80 \%)$ e 5 $(71,40 \%)$ das notificações por essas causas específicas, respectivamente (Tabela 1).

Quanto ao tempo de exposição em que esses imunobiológicos ficaram expostos fora da temperatura preconizada, constatou-se que 38 (79,20\%) das notificações apresentavam tempo de exposição superior a 12 horas e 10 (20,80\%) encontravam-se abaixo de 12 horas (Tabela1). Todos os relatórios $(7,100,00 \%)$ que tinham como causa acondicionamento inadequado, os imunobiológicos permaneceram expostos a temperaturas inadequadas por mais de 12 horas. Dos que apresentaram falha no equipamento e falta de energia como causa, a maior parte teve exposição por um tempo superior a 12 horas apresentando 15 (93,80\%) e 13 (61,90\%), respectivamente. Contudo, $8(38,10 \%)$ dos relatórios referentes à falta de energia também apresentaram tempo de exposição inferior a $12 \mathrm{~h}(\mathrm{p}=0,049)$. 
Id on Line Revista Multidisciplinar e de Psicologia

Id on Line Multidisciplinary and Psycology Journal

Tabela 1. Distribuição proporcional e quantitativa dos fatores determinantes das perdas vacinais segundo zona, temperatura encontrada, tempo de exposição e doses perdidas. Petrolina, 2013.

\begin{tabular}{|c|c|c|c|c|c|c|c|c|c|c|c|c|c|c|}
\hline \multirow{3}{*}{ Determinante da perda } & \multicolumn{4}{|c|}{ Zona* } & \multicolumn{4}{|c|}{ Temperatura encontrada** } & \multicolumn{4}{|c|}{ Tempo de exposição*** } & \multirow{2}{*}{\multicolumn{2}{|c|}{ Total }} \\
\hline & \multicolumn{2}{|c|}{ Urbana } & \multicolumn{2}{|c|}{ Rural } & \multicolumn{2}{|c|}{ Abaixo de $+2^{\circ} \mathrm{C}$} & \multicolumn{2}{|c|}{ Acima de $+8^{\circ} \mathrm{C}$} & \multicolumn{2}{|c|}{$3-12 \mathrm{~h}$} & \multicolumn{2}{|c|}{ Acima de $12 \mathrm{~h}$} & & \\
\hline & $\mathrm{n}$ & $\%$ & $\mathrm{n}$ & $\%$ & $\mathrm{n}$ & $\%$ & $\mathrm{n}$ & $\%$ & $\mathrm{n}$ & $\%$ & $\mathrm{n}$ & $\%$ & $\mathrm{n}$ & $\%$ \\
\hline Falta de energia & 3 & 14,30 & 18 & 85,70 & - & - & 21 & 100,00 & 8 & 38,10 & 13 & 61,90 & 21 & 43,75 \\
\hline Falha no equipamento & 10 & 62,50 & 6 & 37,50 & 5 & 31,30 & 11 & 68,80 & 1 & 6,30 & 15 & 93,80 & 16 & 33,33 \\
\hline $\begin{array}{l}\text { Acondicionamento } \\
\text { inadequado }\end{array}$ & 5 & 71,40 & 2 & 28,60 & 2 & 28,60 & 5 & 71,40 & - & - & 7 & 100,00 & 7 & 14,58 \\
\hline Outros & 2 & 50,00 & 2 & 50,00 & - & - & 4 & 100,00 & 1 & 25,00 & 3 & 75,00 & 4 & 8,33 \\
\hline TOTAL & 20 & $1,70 \%$ & 28 & $58,30 \%$ & 7 & 14,60 & 41 & 85,40 & 10 & 20,80 & 38 & 79,20 & 48 & 100,00 \\
\hline
\end{tabular}

Teste Exato de Fisher. * $\mathrm{p}=0,00333 ; * * \mathrm{p}=0,01677 ; * * * \mathrm{p}=0,049$

Fonte: Elaboração própria a partir dos resultados da pesquisa. 
Id on Line Revista Multidisciplinar e de Psicoloqia

Id on Line Multidisciplinary and Psycology Journal

Quanto ao tempo de exposição dos imunobiológicos fora da temperatura estabelecida a média encontrada foi de 40,25 horas (DP 44,15) variando de um mínimo de 3 horas ao máximo de 216 horas (Tabela 2).

O tempo-resposta da Secretaria Estadual de Saúde de Pernambuco- SES-PE com relação aos resultados da análise dos relatórios dos imunobiológicos que foram colocados sob suspeita apresentaram uma média 45 dias para a devolutiva, variando de 26 a 114 dias de espera (Tabela 2).

Considerando o total de doses perdidas que foi de 10.439 doses, variando de relatório com perda mínima de 9 doses a relatório com perda máxima de 1.009 doses, o valor calculado de vacinas perdidas foi de $\mathrm{R} \$ 72.864,23$ reais, variando de $\mathrm{R} \$ 159,90$ a $\mathrm{R} \$ 8.307,7$ com média de 1.521,40 reais por relatório (Tabela 2).

Tabela 2. Estatística descritiva e intervalo de confiança segundo as variáveis tempo de exposição, tempo- resposta, doses perdidas e valor total de perdas das vacinas. Petrolina-PE, 2013.

\begin{tabular}{lrrrrrr}
\hline \multicolumn{1}{c}{ Variáveis } & Média & Mediana & Mínimo & Máximo & $\begin{array}{r}\text { Desvio } \\
\text { Padrão }\end{array}$ & $\begin{array}{r}\text { Intervalo de } \\
\text { Confiança (95\%) }\end{array}$ \\
\cline { 1 - 5 } $\begin{array}{l}\text { Tempo de exposição } \\
\text { (h*) }\end{array}$ & 40,25 & 24,00 & 3,00 & 216,00 & 44,16 & $27,43-53,07$ \\
Tempo resposta (dias) & 45,90 & 46,00 & 26,00 & 114,00 & 13,97 & $41,84-49,95$ \\
Total de doses perdidas & 217,83 & 176,00 & 9,00 & $1.009,00$ & 207,60 & $157,55-278,11$ \\
$\begin{array}{l}\text { Valor total das perdas } \\
(\mathrm{R} \$)\end{array}$ & $1.521,40$ & $1.234,00$ & 159,90 & $8.307,70$ & $1.593,20$ & $1.058,75-983,96$ \\
\hline
\end{tabular}

Fonte: Elaboração própria a partir dos resultados da pesquisa.

* horas

\section{Discussão}

Segundo o Manual de Rede de Frio, a sensibilidade das vacinas está diretamente relacionada à temperatura de conservação preestabelecida pelo laboratório produtor para manutenção da estabilidade química, física e das propriedades biológicas dos produtos garantindo sua eficácia(BRASIL, 2013). Destacou-se nesse estudo a grande quantidade de doses de vacinas perdidas no município no período da pesquisa, que foi de 10.439 doses, devido 
Id on Line Revista Multidisciplinar e de Psicologia

Id on Line Multidisciplinary and Psycology Journal

a situações onde foram submetidas a temperaturas de conservação distintas daquelas preconizadas pelo Ministério da Saúde (entre $+2{ }^{\circ} \mathrm{C} \mathrm{e}+8^{\circ} \mathrm{C}$ ).

Observou-se que a maioria das vacinas perdidas estavam com temperatura acima do recomendado e estavam relacionadas principalmente a falta de energia elétrica ou falhas no equipamento, comprometendo a potência imunogênica do produto. Da mesma forma aconteceu com a temperatura abaixo do estabelecido, sendo esta em menor proporção levando a perda da potência em caráter permanente. Situação similar encontrada em um estudo realizado no Ceará, onde $75 \%$ das Unidades de saúde analisadas apresentaram registro de temperatura com valores não indicados pelo PNI, ou seja, fora dos valores recomendados entre 2 e 8 graus centígrados(LUNA et al., 2011).

Verificou-se, que todas as perdas ocorridas no município do período do estudo, foram causadas por perdas físicas, ou seja, aquelas ocorridas por exposição à temperatura inadequada, por falta de energia elétrica, falha no equipamento de refrigeração, acondicionamento inapropriado para a conservação das vacinas(BRASIL, 2007b). Esse mesmo resultado foi observado em um estudo realizado no município de Florianópolis(SOARES; OLIVO; SILVA, 2011).

O percentual das ocorrências definidas como "outras causas" apareceram como fator determinante de perdas nos formulários de notificação correspondendo a 8,3\%. Este fator também se caracterizou como perdas físicas, pois incluíam desligamento do disjuntor por ações de vândalos e desligamento acidental da tomada. Resultado distinto do encontrado no estudo de SOARES et al. onde "outras causas" apareceram com $27 \%$, correspondendo a segunda causa de perda(SOARES; OLIVO; SILVA, 2011).

A falta de energia elétrica apareceu como principal fator determinante de perdas apresentando 43,80\%. Esta também foi a principal causa dentre as UBS da zona rural. Tal fato pode estar relacionado com a instabilidade de temperatura em decorrência da distância dessas unidades em relação a zona urbana. $\mathrm{O}$ acondicionamento inadequado das vacinas esteve mais presente na zona urbana. Este pode está diretamente relacionado a atuação profissional em sala de vacina o que pode comprometer o cumprimento dos requisitos estabelecidos para conservação das mesmas.

Um estudo realizado com profissionais que atuam em imunização em Minas Gerais apontou falhas na estrutura das salas de vacina, deficiência no processo de supervisão, 
Id on Line Revista Multidisciplinar e de Psicologia

Id on Line Multidisciplinary and Psycology Journal

desconhecimento das normas técnicas do PNI e ausência de educação para os trabalhadores(OLIVEIRA et al., 2015). Tais fatores podem explicar a presença ainda de causas das perdas das vacinas por acondicionamento inadequado.

O presente estudo difere dos achados da pesquisa realizada em Florianópolis que teve como fator principal a falha no equipamento de refrigeração (SOARES; OLIVO; SILVA, 2011). O Ministério da Saúde orienta que havendo interrupção no fornecimento de energia elétrica, o ideal é manter o equipamento fechado e monitorização rigorosa da temperatura interna. Quando a temperatura estiver próxima a $7^{\circ} \mathrm{C}$, proceder imediatamente a transferência dos imunobiológicos para outro equipamento (refrigerador ou caixa térmica) com temperatura recomendada (BRASIL, 2013).

Da mesma forma deve-se proceder nos casos de falha de equipamento de refrigeração. Pesquisa realizada na cidade do Recife identificou que 46,1\% dos equipamentos de refrigeração apresentaram problemas como geladeiras com inadequada condição de vedação(MELO; OLIVEIRA; ANDRADE, 2010).

Segundo o Manual da Rede de Frio, faz-se necessária a realização de revisão periódica do equipamento de refrigeração, responsáveis pela conservação das vacinas, com o objetivo de se verificar qualquer tipo de falha(BRASIL, 2001b).

A geladeira de uso doméstico é o equipamento de uso para refrigeração dos produtos nas unidades de saúde. São destinadas à estocagem de imunobiológicos em temperaturas $\mathrm{a}+2^{\circ} \mathrm{C}$ $\mathrm{e}+8^{\circ} \mathrm{C}$, devendo para isto estar reguladas para funcionar nesta faixa de temperatura(BRASIL, 2007b).

Os principais cuidados para manutenção desses equipamentos prevenindo assim falhas evitáveis incluem o controle da temperatura por meio do termômetro diariamente. Antes de iniciar as atividades e ao final do dia, deve-se fazer o registro da temperatura. $\mathrm{O}$ equipamento deve estar conectado em tomada exclusiva. Se houver mais de uma geladeira, fazer o degelo a cada 15 dias ou quando a camada de gelo for superior a $0,5 \mathrm{~cm}$. Além disso, não colocar qualquer elemento na geladeira que dificulte a circulação de ar e verificar a vedação da borracha e o nivelamento para regulagem da porta, para evitar que ela fique entreaberta(BRASIL, 2007b).

O que se observou na análise dos relatórios foi que a maioria das interrupções de energia acontecia no fim de semana ou à noite, horários que não havia funcionamento das unidades de 
Id on Line Revista Multidisciplinar e de Psicologia

Id on Line Multidisciplinary and Psycology Journal

saúde, dessa forma o tempo de exposição a temperaturas inadequadas ocorriam em longos períodos.

O tempo médio de exposição das vacinas fora da temperatura estabelecida foi de 40,25 horas. O PNI estabelece que caso o imunobiológico tenha sido exposto a temperatura igual ou acima de $25^{\circ} \mathrm{C}$ (vinte e cinco) por período superior a 24 (vinte e quatro) horas o produto deve ser descartado (BRASIL, 2013).

Quando há evidência comprovada de que a vacina foi submetida a uma variação da temperatura de conservação, diferente da preconizada (entre $+2{ }^{\circ} \mathrm{C} \mathrm{e}+8{ }^{\circ} \mathrm{C}$ ), o Ministério da Saúde orienta determinados procedimentos específicos para análise da estabilidade das vacinas utilizadas nos programas de imunização, destacando situações de exposição a altas temperaturas e a temperaturas de congelamento (BRASIL, 2013).

Assim, nas situações em que o imunobiológico tenha sido submetido à temperatura de conservação distinta da preconizada, verifica-se a indicação de avaliação, mediante análise do registro de ocorrência referido no Formulário de Registro de Ocorrência de Alterações Diversas (BRASIL, 2013).

O Formulário deve ser preenchido pelo responsável da Unidade de saúde e encaminhado ao PNI municipal, que por sua vez encaminhará esse formulário de notificação ao PNI da Região de Saúde onde ficará responsável de enviar aos técnicos do nível central da Secretaria Estadual de Saúde de Pernambuco- SES-PE(BRASIL, 2013). Após avaliação preliminar, o mesmo será encaminhado pela Coordenação Estadual à Coordenação Geral do Programa Nacional de Imunizações (CGPNI) o mais rápido possível, para análise das informações e definição de conduta (BRASIL, 2007b).

Analisando os resultados, o tempo médio da resposta da SES-PE sobre as vacinas colocadas sob suspeita foi de 45 dias, chegando a variações entre 26 e 114 dias de espera. Segundo o Manual de Vacinação, quando um imunobiológico é colocado sob suspeita deve ser submetido a processos de análise e/ou reteste. É por meio da análise das informações contidas no Formulário de Registro de Ocorrência que a CGPNI avaliará a situação de suspeita recomendando ou não o reteste, ou indicando a autorização para utilização ou descarte do imunobiológico. O tempo de duração do reteste varia entre 45 e 90 dias, dependendo do imunobiológico. As vacinas avaliadas não passaram pelo processo de reteste, foram indicados seus descartes. 
Id on Line Revista Multidisciplinar e de Psicologia

Id on Line Multidisciplinary and Psycology Journal

\section{Conclusão}

Em vista dos resultados encontrados, a zona rural apresentou o maior número de relatórios de perdas, a falta de energia apareceu como principal causa determinante das perdas das vacinas acometendo principalmente a zona rural de localização das UBS. O tempo de exposição desses imunobiológicos foi superior a $12 \mathrm{~h}$ tendo sido expostos a temperaturas superiores ao que é preconizado. Contudo, apesar do acondicionamento inadequado das vacinas ter sido a terceira causa, este foi mais presente na zona urbana. Tal fato direciona para a atuação da equipe profissional frente aos cuidados na conservação das vacinas que são utilizadas na Atenção Primária a Saúde.

A realização de estudos afins ao tema exposto também é de grande relevância, principalmente em relação ao custo- efetividade para se avaliar os gastos com as perdas, comparando a gastos com a manutenção preventiva de toda rede, como exemplo, a substituição dos equipamentos de refrigeração. Assim, as despesas causadas com as perdas das vacinas, poderiam ser investidas na ampliação dos serviços de saúde.

É importante investir em capacitação permanente dos profissionais que atuam nas salas de vacinas, para que esses possam identificar qualquer alteração que venha acontecer que interfiram na conservação dos imunobiológicos e diminua sua eficácia. Portanto, garantir a qualidade das vacinas que são ofertadas nos serviços de saúde à população, contribuem para o controle das doenças imunopreveníves. Espera-se que esse estudo possibilite subsidiar os gestores na estruturação das salas de vacina e assim poder contribuir no controle das doenças imunopreveníveis.

A escassez de estudos enfatizando os fatores determinantes nas perdas de vacina ocasionou dificuldade na discussão dos resultados encontrados. Assim, novos estudos devem ser realizados a fim de complementar os resultados encontrados na presente pesquisa.

\section{Referências}

BRASIL. Ministério da Saúde. Manual de normas de vacinação. 3. ed. Brasília: Ministério da Saúde: Fundação Nacional de Saúde, 2001a. 
Id on Line Revista Multidisciplinar e de Psicologia

Id on Line Multidisciplinary and Psycology Journal

BRASIL. Ministério da Saúde. Secretaria de vigilância em Saúde. Manual de Rede de Frio. 4. ed. Brasília: Ministério da Saúde, 2007a.

BRASIL. Ministério da Saúde. Fundação nacional de Saúde. Programa Nacional de Imunizações. Manual de Rede de Frio. 3. ed. Brasília: Ministério da Saúde, 2001b.

BRASIL. Ministério da Saúde. Secretaria de Vigilância em Saúde. Departamento de Vigilância Epidemiológica. Manual de Rede de Frio do Programa Nacional de Imunização. Departamento de Vigilância Epidemiológica. 4. ed. Brasília: Ministério da Saúde, 2013.

BRASIL. Ministério da Saúde. Secretaria Nacional de Ações Básicas de Saúde. Programa Nacional de Imunizações. Manual para determinação e reparo de avarias em refrigeradores/congeladores fotovoltaicos para a conservação de vacinas. Brasília: Centro de Documentação do Ministério da Saúde, 2007.

CUNHA, J; KREBS, L.S.; BARROS, E. Vacinas e imunoglobulinas: consulta rápida. Porto Alegre: Artmed, 2009.

LUNA, Geisy Lanne Muniz et al . Aspectos relacionados à administração e conservação de vacinas em centros de saúde no Nordeste do Brasil. Ciênc. saúde coletiva, Rio de Janeiro , v. 16, n. 2, p. 513521, Feb. 2011 . Disponível em: <http://www.scielo.br/scielo.php?script=sci_arttext\&pid=S141381232011000200014\&lng=en\&nrm=iso>. Acesso em: 13 Nov. 2016.

MELO, Giselle Karine Muniz de; OLIVEIRA, Janice Vasconcelos; ANDRADE, Maria Sandra. Aspectos relacionados à conservação de vacinas nas unidades básicas de saúde da cidade do Recife Pernambuco. Epidemiol. Serv. Saúde, Brasília, v. 19, n. 1, p. 26-33, mar. 2010. Disponível em $<$ http://scielo.iec.pa.gov.br/scielo.php?script=sci_arttext\&pid=S1679-

$49742010000100004 \& \operatorname{lng}=$ pt\&nrm=iso>. acessos em 09 nov. 2016.

OLIVEIRA, Valéria Conceição de et al . Fragilidades da conservação de vacina nas Unidades de Atenção Primária à Saúde. Rev. Bras. Enferm., Brasília , v. 68, n. 2, p. 291-296, abr. 2015. Disponível em $<$ http://www.scielo.br/scielo.php?script=sci_arttext\&pid=S003471672015000200291\&lng=pt\&nrm=iso>. Acesso em: 13 nov. 2016.

SOARES, K.R.A.; OLIVO, L.C.C.; SILVA, M.L.B. Ações para eliminar as perdas físicas de vacinas no município de Florianópolis. Coleção Gestão da Saúde Pública , 2011, v. 9, p. 93-103.

VICARE, C.F.S. Eventos Adversos Pós-Vacinação em Crianças no Estado de Santa Catarina. Florianópolis: UFSC, 2008.

\section{Como citar este artigo (Formato ABNT):}

SANTOS, J.A.S.; TORRES, D.B.; MELO, R.A.; FERNANDES, F.E.C.V.; CAMPOS, M.E.A.L. Fatores determinantes das perdas das vacinas em Unidades Básicas de Saúde. Id on Line Revista Multidisciplinar e de Psicologia, Nov-Dez. de 2016, vol.10, n.32, p. 41-53. ISSN: 1981-1179.

Recebido: $14 / 11 / 2016$

Aceito: $16 / 11 / 2016$ 